Draft Version July 10, 2004

Preprint typeset using $\mathrm{LAT}_{\mathrm{E}} \mathrm{X}$ style emulateapj v. 4/12/04

\title{
RECONSTRUCTING AND DECONSTRUCTING DARK ENERGY
}

\author{
ERIC V. LINDER \\ Physics Division, Lawrence Berkeley Laboratory, Berkeley, CA 94720 \\ Draft version July 10, 2004
}

\begin{abstract}
The acceleration of the expansion of the universe, ascribed to a dark energy, is one of the most intriguing discoveries in science. In addition to precise, systematics controlled data, clear, robust interpretation of the observations is required to reveal the nature of dark energy. Even for the simplest question: is the data consistent with the cosmological constant? there are important subtleties in the reconstruction of the dark energy properties. We discuss the roles of analysis both in terms of the Hubble expansion rate or dark energy density $\rho_{D E}(z)$ and in terms of the dark energy equation of state $w(z)$, arguing that each has its carefully defined place. Fitting the density is best for learning about the density, but using it to probe the equation of state can lead to instability and bias.

Subject headings: dark energy — cosmology:observations — cosmology:theory
\end{abstract}

\section{INTRODUCTION}

The acceleration of the expansion of the universe represents a challenge to our understanding of fundamental physics. Whether the resolution of this mystery lies in high energy physics, the theory of gravitation, the nature of the quantum vacuum, extra dimensions, etc. remains unknown. The simplest model, and possibly one motivated from string theory (Kachru 2003), is Einstein's cosmological constant, though it too is fraught with complications, e.g. the fine tuning and coincidence problems (Weinberg 1989; Carroll 2001).

Nevertheless, the cosmological constant serves as a benchmark, a first indication for how drastically we might need to extend our physics framework to understand the acceleration of the universe. Additionally, because this model offers such definite and unvarying predictions for the dark energy properties, it gives a robust target. In this paper we consider various aspects of interpretation of the data, present and future, that aid or obscure the goal of determining the nature of dark energy and its consistency with the cosmological constant. In the sense that conflicting or biased interpretations of the data, rather than the data itself, affect what we determine to be the reality, we refer to this problem as "deconstruction" of dark energy.

\section{RECONSTRUCTION AND DECONSTRUCTION}

The cosmological constant has an energy density that is constant in time, $\rho(z)=\rho(0)$ in terms of redshift $z$, a pressure the negative of the energy density, $p=-\rho$, or equivalently an equation of state ratio (EOS) $w \equiv p / \rho=$ -1 constant in time, and no spatial fluctuations in $\rho$ or $w$. So we could think of testing whether the dark energy is a cosmological constant by seeing if the observations are consistent with either a constant density or $w=-1$.

\subsection{Constant equation of state $w_{\text {const }}=-1$}

The first point to make is that fitting the data in terms of a constant EOS parameter is insufficient. Many models with time variation $w^{\prime}$ can be fit, for data of limited precision or over a limited redshift range, by a $w_{\text {const }}$.

Electronic address: evlinder@lbl.gov
But this obscures the physics behind the dark energy. Even if we are so modest and undemanding as to only ask if $w_{\text {const }}=-1$ or not, we can be fooled by time varying models mimicking this value.

We illustrate this in Figure [1] The two dark energy models shown, one a high energy physics model parametrized with a time evolution $w(a)=w_{0}+w_{a}(1-$ $a)$, where $a=1 /(1+z)$ is the scale factor of the universe, with $\left(w_{0}, w_{a}\right)=(1.5,-1.5)$, and one an extended gravity model (case 3 of Linder 2004) modifying the Friedmann expansion equation, both possess a strong time variation in the equation of state. However, in comparison to present data both appear consistent with a cosmological constant when interpreted in terms of a $w_{\text {const }}$.

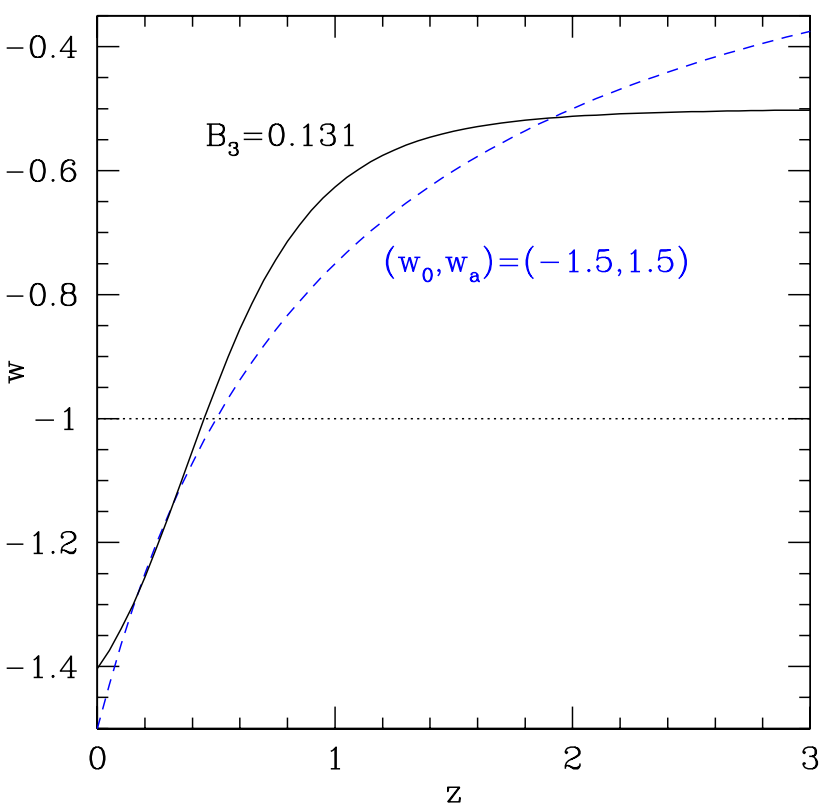

FIG. 1. - Dark energy models possessing strong time variation can still appear consistent with cosmological constant when interpreted only in terms of a constant equation of state. The two models shown here are consistent with all current data and a $w_{\text {const }}=-1$ cosmological constant model. 
Both models are consistent, to $0.1 \%$, with the distance to the last scattering surface of the cosmic microwave background $(\mathrm{CMB})$ in the cosmological constant model with the same matter density. Even the next generation Planck experiment (see Planck website) will not be able to distinguish these models from the cosmological constant on this basis. With regard to large scale structure, the linear theory growth factors agree to better than $4 \%$. This roughly corresponds to requiring knowledge of the mass fluctuation amplitude $\sigma_{8}$ at this level, but the current systematic uncertainties appear to be of order $20 \%$. Most constraining are the Type Ia supernova distances, for which the time varying models deviate from the cosmological constant by under 0.1 magnitude. This is currently at the $1 \sigma$ limit of precision.

So if our universe followed either of these dark energy models (or a host of others that could be chosen) then by using the $w_{\text {const }}$ paradigm we would happily interpret the data as pointing to a cosmological constant and miss the key clues to new physics.

Of course as cosmological probes become more precise, we will be able to distinguish the illustrated models from the cosmological constant. However if the interpretation does not include the possibility of time variation in the dark energy equation of state we will again miss the physics and think the particular models shown are $w_{\text {const }}=-1.2$ models. And indeed dark energy models with a less extreme time variation than shown in Fig. 1 might well look like $w_{\text {const }}=-1$ to within the data precision. Moreover, many models have an asymptotic or attractor behavior that brings the EOS very near the cosmological constant value for a period of the expansion history. Examples include the linear potential model (Linde 1987; Kallosh et al. 2003), the cyclic model (Steinhardt \& Turok 2004), the "ripstop" model where particle production offsets the superacceleration of phantom energy with $w<-1$ (Linder 2004), and certain scalar-tensor theories (Matarrese et al. 2004).

For a window onto the new physics we need to look for both the present or averaged EOS value and a measure of its time variation, say $w^{\prime}$. Note that throughout this paper we refer to the EOS function $w(z)$ as an effective EOS, defined in terms of the expansion history $a(t)$, and not necessarily restricted to a physical component like a scalar field. From Linder \& Jenkins 2003 (cf. Saini et al. 2000) we have the effective EOS defined by

$$
w(z)=-1+\frac{1}{3} \frac{d \ln \delta H^{2}}{d \ln (1+z)},
$$

where the Friedmann expansion equation reads

$$
H^{2}(z) / H_{0}^{2}=\Omega_{M}(1+z)^{3}+\delta H^{2}(z) .
$$

The Hubble parameter $H=\dot{a} / a$ and $\Omega_{M}$ is the matter density, so $\delta H^{2}$ represents our ignorance of either components beyond matter or of extensions to general relativity that modify the Friedmann equation.

A very robust and convenient measure of the time variation of the dark energy EOS is given by the two parameter form (Linder 2003; Linder 2004)

$$
w(z)=w_{0}+w_{a}(1-a)=w_{0}+w_{a} z /(1+z) .
$$

We see that this implies a characteristic variation

$$
\partial_{N} w \equiv-\frac{d w}{d \ln a}=a w_{a} .
$$

Note that this is closely related to the inflationary theory measure of time variation:

$$
\partial_{N} w=-d w / d N,
$$

where $N=d \ln a$ counts the number of e-folds of expansion during inflation. Just as in inflation, there is "running" - a variation of parameters during the acceleration - and we need to choose when to evaluate the derivatives, e.g. at $N=60$. The derivative of the EOS will also be a function of the redshift when it is evaluated, and for simplicity we define a number from the function: $w^{\prime} \equiv \partial_{N} w(z=1)=w_{a} / 2$. This is motivated by $z \approx 1$ being approximately when dark energy begins to become dynamically important for the expansion.

So we have argued that to learn the direction of new physics we need to consider not only the value of $w_{\text {const }}$ but not deny the possibility of time variation $w^{\prime}$. That is, if we want to say that observations point to the cosmological constant, we need to see that $w^{\prime} \approx 0$. A much more modest goal is merely to ask whether the observations are consistent with the cosmological constant. In this case, we might consider looking for whether the dark energy density is constant, rather than addressing the physics question of its equation of state.

\subsection{Constant density}

Note that reconstructing a constant energy density is only a necessary, not sufficient, condition for a cosmological constant. Some models might arrange an effective energy density nearly constant with time yet with effective pressure not equal to the negative of the energy density; this holds in some k-essence and tachyon field models (cf. Scherrer 2004). So mapping the density alone contains insufficient information if we want to remain open to the possibility that the unknown physics could involve components other than canonical, minimally coupled scalar fields or involve extensions to gravity.

But in asking for consistency, not insight, seeking to reconstruct the dark energy density behavior (see e.g. Wang \& Tegmark 2004; Wang \& Freese 2004; Huterer \& Coorav 2004) is a perfectly acceptable method. Testing for the EOS $w(z)$ also allows one to check consistency (plus more of course), and one can also build up the density history through an integration:

$$
\rho(z)=\rho(0) e^{3 \int_{0}^{z} d z^{\prime}\left[1+w\left(z^{\prime}\right)\right] /\left(1+z^{\prime}\right)} .
$$

Of course this will diminish the sensitivity of the reconstruction of $\rho(z)$ somewhat, though not drastically (Huterer \& Coorav 2004). Also, it may be easier to test for a specific value, e.g. $w=-1$, than for a property, e.g. $\rho=$ constant (Huterer 2004). Generally, if one is only interested in whether the density is constant, use reconstruction in terms of the density (though in terms of EOS is not unreasonable). However, if one is interested in the physics behind the dark energy, the EOS reconstruction is strongly preferred.

This is because going from $\rho(z)$ to $w(z)$ requires differentiation, whether explicitly or implicitly, and this leads to instability and bias in the results. This is what we referred to in the introduction as the deconstruction problem. The dangers of explicit differentiation of the data are well known (Huterer \& Turner 1999; Weller \& Albrecht 2002; Tegmark 2002), but those of 
implicit differentiation have been illustrated more recently. Jönsson et al. 2004 pointed out that assuming a functional form for the density, or Hubble parameter, could distort conclusions regarding the equation of state, even apparently preferring a time evolving dark energy to fit data of a cosmological constant universe.

Here we extend this to the general translation from a density reconstruction to an equation of state behavior. Consider a set of values $\left\{\rho_{i}\right\}$, representing the density in redshift bins centered at $z_{i}$. To obtain the EOS, one can imagine fitting the reconstructed $\rho_{i}$ with a polynomial or spline interpolation $\rho(z)$ (cf. Wang \& Tegmark 2004) and then carrying out the derivative to form

$$
w(z)=-1+\frac{1}{3 \rho}(1+z) \frac{d \rho}{d z} .
$$

In terms of the binned values,

$$
\begin{aligned}
w(z)=-1+ & \frac{1+z}{3 \rho_{i}}\left\{\frac{\rho_{i+1}-\rho_{i}}{z_{i+1}-z_{i}}\right. \\
& -\frac{z_{i+1}-z_{i}}{6} \rho_{i}^{\prime \prime}\left[\frac{3\left(z_{i+1}-z\right)^{2}}{\left(z_{i+1}-z_{i}\right)^{2}}-1\right] \\
& \left.+\frac{z_{i+1}-z_{i}}{6} \rho_{i+1}^{\prime \prime}\left[\frac{3\left(z-z_{i}\right)^{2}}{\left(z_{i+1}-z_{i}\right)^{2}}-1\right]\right\},
\end{aligned}
$$

where the second derivatives are determined in terms of the free parameters (bin values $\rho_{i}$ ) through the cubic spline formula and boundary conditions. Thus we can write Eq. 8 illustratively as

$$
w(z)=-1+[1 /(3 \rho)](1+z) \sum c_{i} \rho_{i},
$$

where $\rho_{i}$ are the free parameters of the problem and $c_{i}$ are weight functions. One can imagine these being more general than cubic spline coefficients and instead being either polynomial expansions in redshift, e.g. $c_{i}=b_{i}\left(z-z_{i}\right)^{n}$, or involving orthogonal basis functions, so the following conclusions should be fairly general.

Following the method of Jönsson et al. 2004, now consider the stability of this deconstruction to small perturbations $\delta \rho_{i}$. The quantity in braces in Eq. 8 (or its generalizations) will involve terms like $\delta \rho_{i},\left(\partial \rho_{i}^{\prime \prime} / \partial \rho_{i}\right) \delta \rho_{i} \sim$ $\delta \rho_{i}$. However, formally, because of the $1+z$ factor in front of the braces, a small perturbation to the fit to the data in terms of $\rho_{i}$ will run away at high redshift in terms of $w(z)$, showing the instability of this approach ${ }^{1}$. (Note that redshifts not much higher than 1 may be sufficient; Jönsson et al. 2004 find instability regions where $|w|>15$ for $z<1.8$.)

Furthermore, this approach not only mediates against the cosmological constant (or any $w_{\text {const }}$ model), but favors a model where the EOS crosses from less than -1 to greater than -1 : precisely what is seen in current reconstructions. This occurs because the quantity in braces is redshift dependent and will have a zero at some redshift $z_{*}$. That is, for generic sets $\left\{\rho_{i}\right\}$ one will find $w\left(z_{*}\right)=-1$ (not necessarily within the redshift range of the data). Thus the deconstruction legislates for crossing the value

\footnotetext{
1 It also may be problematic to take a linear interpolation as one of the spline boundary conditions as Wang \& Tegmark 2004 do. (Note that the $\rho^{\prime}(z=0)=\rho\left(z_{1}\right) / z_{1}$ appearing there is a typo for $\rho^{\prime}(z=0)=\left[\rho\left(z_{1}\right)-\rho(0)\right] / z_{1}$.) This may allow statistical excursions of the density off the cosmological constant behavior to cause $w \neq-1$.
}

-1 . Combining these two effects together, a small perturbation $\delta \rho_{i}$ will generate a larger $\delta w$, forcing $w$ off from -1 (if the cosmological constant were the true fit) and forcing $w$ to cross -1 - both leading to evolution.

In summary, using $\rho_{i}$ fitting to find $\rho(z)$ is fine, but using $\rho_{i}$ to discuss $w(z)$ is perilous. The latter generates errors of two types: false negatives where a true cosmological constant appears not to be so, and false positives where any model looks, over some finite redshift interval, not very distinct from a cosmological constant!

That said, there appears to be a simple, if partial fix. We can rewrite Eq. 7 in terms of a logarithmic derivative with respect to scale factor $a$, rather than $1+z$. If bins are defined in terms of $\rho(a)$, then the high redshift secular instability does not appear, and this should mitigate any tendency to cross the value -1 . General issues of numerical differentiation, bin to bin, instability and the need for careful treatment of spline boundary conditions remain, but are possibly less severe.

\subsection{Density or Equation of State?}

What about the breadth of models encompassed by the two approaches? It has sometimes been claimed (Wang \& Tegmark 2004) that $w(z)$ suffers from not covering more esoteric possibilities like negative density. This is not wholly true, and even so is more of a feature than a bug. From Eq. 1 one sees that negative densities can be handled straightforwardly in terms of $w(z)$. For example, consider a model where we (mistakenly) think $\Omega_{M}=1.2$ and find the best fit to data gives $\delta H^{2}=-0.2(1+z)^{3}$. Clearly this looks like a negative density. But $\left[(1+z) / \delta H^{2}\right] d \delta H^{2} / d(1+z)$ is well defined and gives $w(z)=0$ : exactly as expected for this extra (negative) matter component.

Where the $w(z)$ parametrization does blow up is not for negative density but for density crossing through zero. In the parametrization of Wang \& Tegmark 2004, they normalize the density by the present value, using $X(z)=$ $\rho(z) / \rho(0)$, with of course $X(0)=1$. In the negative density illustration above, $X$ will then always be positive. It is only when $X$ goes from its defined value of 1 at $z=0$ to 0 at some redshift $z_{*}$ that $w\left(z_{*}\right)$ blows up to infinity. But we can argue this is a feature not a bug.

For example, in the linear potential model analyzed in Kallosh et al. 2003, the dark energy density hits zero very shortly in cosmic time before the final "cosmic doomsday" collapse. By parametrizing the $w(z)$ given by the linear potential (e.g. in terms of $w_{0}$ and $w_{a}$, contrary to the claim by Wang \& Tegmark 2004 that this model cannot treat the collapse case) and asking when it blows up (in the future), one obtains a very accurate estimate for the doomsday time.

Furthermore, having $\rho$ cross through zero is not merely mildly esoteric, but violates either the Big Bang condition or the continuity equation

$$
d \rho / d \ln (1+z)=3(\rho+p) .
$$

Dividing through by $\rho(0)$, one has $d X / d \ln (1+z)=3(X+$ $p / \rho(0))$. For acceleration, we take the pressure $p$ to be negative and so at the time when $X=0$, the left hand side will be negative and $\rho$ will be driven further negative at high redshift. This negative energy density will cause the expansion rate $H$ to go to zero at some point in the past, describing a bounce universe rather than a Big 
Bang model. If one insists on allowing the density to cross through zero then the price is either abandoning the continuity equation or the Big Bang early universe. And if one abandons the continuity equation then the density alone is insufficient since pressure is unknown, leading back to an equation of state formulation.

Finally, one can argue that the Friedmann equation of motion explicitly involves both energy density $\rho$ and pressure $p$, so a parametrization $w(z)=p(z) / \rho(z)$ is closer to the physics. An example of this was pointed out at the beginning of 2.2 where a constant energy density may not necessarily be matched by a constant negative pressure of equal magnitude. Fundamentally, the passive gravitational energy $\rho+p$ and the active gravitational energy $\rho+3 p$ both explicitly depend on more than merely the density, they involve an equation of state.

\section{CONCLUSION}

Understanding, or at least obtaining insight into, the nature of dark energy will be the great challenge of physics in the next decade. We must be sure that we ask questions in such a way that the answers we derive are not deconstructions - subjective interpretations - but faithful reconstructions of aspects of the true physics. This includes allowing explicitly for the possibility of time variation in the equation of state of the dark energy. Using a $w_{\text {const }}$ will only tell us whether the data are consistent with the cosmological constant, not teach us that another, mimicking model is not the true answer.
If our main concern is whether the dark energy density is constant in time, we can use reconstruction of the density values in (preferably uncorrelated) bins in expansion factor or a principal component analysis of the density (see Huterer \& Coorav 2004; Huterer \& Starkman 2003). Using the equation of state $w(z)$ in either bins or eigenmodes will give almost as accurate results, plus additional information. Crosschecking one versus the other may also test exotic models involving violation of the continuity equation.

In our quest to understand new physics, key clues are carried by the equation of state. To reveal this one should fit for $w(z)$ directly. Using other, intermediary parametrizations such as density on the way to the equation of state can lead to unstable and biased solutions, both type 1 (false positive) and type 2 (false negative) errors with respect to determining if the data are consistent with no time evolution, $w(z)=-1$, like for the cosmological constant model. Of course systematics errors in the data themselves can also lead to false evolution.

\section{ACKNOWLEDGMENTS}

This work has been supported in part by the Director, Office of Science, Department of Energy under grant DE-AC03-76SF00098. I thank Alex Kim for useful comments, and especially the Michigan Center for Theoretical Physics for hospitality during the writing of this paper and Katie Freese, Paolo Gondolo, and Yun Wang for discussions there.

\section{REFERENCES}

S.M. Carroll, Living Rev. Rel. 4, 1 (2001) astro-ph/0004075

D. Huterer, private communication

D. Huterer and A. Cooray, astro-ph/0404062

D. Huterer and G. Starkman, Phys. Rev. Lett. 90, 031301 (2003) astro-ph/0207517

D. Huterer and M.S. Turner, Phys. Rev. D 60, 081301 (1999) astro-ph/9808133

J. Jonsson, A. Goobar, R. Amanullah, and L. Bergström, astro-ph/0404468

S. Kachru, R. Kallosh, A. Linde, and S.P. Trivedi, Phys. Rev. D 68, 046005 (2003) hep-th/0301240

R. Kallosh, J. Kratochvil, A. Linde, E.V. Linder, and M. Shmakova, JCAP 0310, 15 (2003) astro-ph/0307185

A.D. Linde, in Three hundred years of gravitation, eds. S.W. Hawking and W. Israel, p. 604 (1987)

E.V. Linder, Phys. Rev. Lett. 90, 091301 (2003) astro-ph/0208512

E.V. Linder, accepted to Phys. Rev. D astro-ph/0402503
E.V. Linder and A. Jenkins, MNRAS 346, 573 (2003) astro-ph/0305286

S. Matarrese, C. Baccigalupi, and F. Perrotta, astro-ph/0403480 Planck: http://astro.estec.esa.nl/planck

T.D. Saini, S. Raychaudhury, V. Sahni, and A.A. Starobinsky, Phys. Rev. Lett. 85, 1162 (2000) astro-ph/9910231

R.J. Scherrer, accepted to Phys. Rev. Lett., astro-ph/0402316

P.J. Steinhardt and N. Turok, astro-ph/0404480

M. Tegmark, Phys. Rev. D 66, 103507 (2002) astro-ph/0101354

Y. Wang \& K. Freese, astro-ph/0402208

Y. Wang and M. Tegmark, accepted to Phys. Rev. Lett., astro-ph/0403292

S. Weinberg, Rev. Mod. Phys. 61, 1 (1989)

J. Weller and A. Albrecht, Phys. Rev. D 65, 103512 (2002) astro-ph/0106079 